\title{
Short-Time Events, Coherence, and Structural Dynamics in Photochemistry of Aqueous Halogenated Transition Metal Dianions
}

\author{
A. N. Tarnovsky ${ }^{1}$, I. L. Zheldakov ${ }^{2}$, P. Z. El-Khoury ${ }^{3}$, S. K. Pal ${ }^{4}$, A. S. Mereshchenko ${ }^{1}$, M. N.

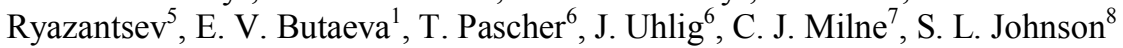 \\ ${ }^{1}$ Department of Chemistry, Center for Photochemical Sciences, Bowling Green State University, \\ Bowling Green, OH, USA \\ ${ }^{2}$ Department of Chemistry, University of Kansas, Lawrence, KS 66045, USA \\ ${ }^{3}$ Department of Chemistry, University of California, Irvine, California 92697, USA \\ ${ }^{4}$ School of Basic Sciences, Indian Institute of Technology, Mandi, Himachal Pradesh 175 001, India \\ ${ }^{5}$ Department of Chemistry, Emory University, Atlanta, GA 30322, USA \\ ${ }^{6}$ Department of Chemical Physics, Lund University, Lund, 22100 Sweden \\ ${ }^{7}$ Laboratory of Ultrafast Spectroscopy, EPFL, Lausanne, CH-1015 Switzerland \\ ${ }^{8}$ Institut für Quantenelektronik, Eidgenössische Technische Hochschule Zürich, Zürich CH-8093, \\ Switzerland
}

\begin{abstract}
Ultrafast pump-probe spectroscopy, time-resolved x-ray absorption, and computational photochemistry elucidate the photochemical pathway of hexabromoplatinate dianions that propagates through distortions of nascent pentabromoplatinate anions caused by Jahn-Teller conical intersections and terminates at aquated product complexes.
\end{abstract}

Photoprocesses in hexahalo dianions have been discussed invoking two distinct mechanisms in which the complex relaxes from the initially excited electronic state to either the vibrationally excited ground-electronic state or lower-lying electronic excited state, followed by metal-halogen bond breaking, [1]. In aqueous environments, resulting polyatomic photofragments may undergo subsequent aquation.

In this work, aqueous $\mathrm{PtBr}_{6}{ }^{2-}$ was excited into the mixed ${ }^{1} \mathrm{~T}_{2 \mathrm{~g}}$ ligand-field/charge-transfer state (LF/CT) and the dissociative lowest excited triplet ${ }^{3} \mathrm{~T}_{1 \mathrm{~g}} \mathrm{LF}$ state at $420 \mathrm{~nm}$ and $530 \mathrm{~nm}$, respectively. The photochemical reaction path involves separation of negative charges $\left(\mathrm{PtBr}_{6}{ }^{2-} \rightarrow \mathrm{PtBr}_{5}{ }^{-}+\mathrm{Br}^{-}\right)$, distortions of a nascent penta-bromoplatinate anion caused by a Jahn-Teller (JT) $\mathrm{C}_{4 \mathrm{v}}$ conical intersection $(\mathrm{CI})$, and hydration to form $\mathrm{PtBr}_{5}\left(\mathrm{H}_{2} \mathrm{O}\right)^{-}$. To characterize the reaction pathways, intermediates and products, we used ultrafast pump-probe and time-resolved x-ray absorption spectroscopies, in concert with CASPT2 and DFT/TDDFT calculations. The assignment of the UVvis spectrum of aqueous $\mathrm{PtBr}_{6}^{2-}$ has been carried out using CASPT2 calculations of vertical electronic transitions (VETs), [2]. The results were reproduced using the less computationally expensive TDDFT/M052X method in combination with a conductor-like polarizable continuum model (CPCM). The VET data for possible products from the later method were used for spectral assignments of transient absorption $\triangle \mathrm{A}$ spectra. The ${ }^{1} \mathrm{~T}_{1 \mathrm{~g}} \mathrm{LF} / \mathrm{CT}$ state and the dissociative ${ }^{3} \mathrm{~T}_{1 \mathrm{~g}} \mathrm{LF}$ state of $\mathrm{PtBr}_{6}{ }^{2-}$ both give rise to the same 500-nm absorption band, Fig. 1. The growth of this band 
occurs with a 150-fs time constant following 420-nm excitation and is assigned to the formation of triplet ${ }^{3} \mathrm{PtBr}_{5}{ }^{-}$in the nearly trigonal bipyramid geometry $\left(\mathrm{C}_{2 \mathrm{v}}\right)$. This indicates remarkably fast intersystem crossing and dissociation of the parent $\mathrm{PtBr}_{6}{ }^{2-}$ complex, and suggests that the previous interpretation of the primary photo-processes in $\mathrm{PtBr}_{6}{ }^{2-}$ is incorrect, [3]. Coherent oscillations (frequencies, 78 and $114 \mathrm{~cm}^{-1}$ ) are observed within the $500-\mathrm{nm}$ absorption band of ${ }^{3} \mathrm{PtBr}_{5}{ }^{-}$. These beats are weak near the center, but strong at the blue and red wings of the $500-\mathrm{nm}$ band, where they exhibit a $\pi$ phase shift. Spectral narrowing and blue shift of the $500-\mathrm{nm}$ band $(600 \mathrm{fs})$ is followed by its decay and development of the broad $430-\mathrm{nm}$ band $(\sim 1.6 \mathrm{ps})$ assigned to ${ }^{1} \mathrm{PtBr}_{5}^{-}$, indicating intersystem crossing in ${ }^{3} \mathrm{PtBr}_{5}{ }^{-}$. The 430 -nm band decays on two time scales with lifetimes of $\sim 7$ and $\sim 15 \mathrm{ps}$, which is accompanied by the partial recovery and subsequent growth of ground-state bleach. These processes are assigned, respectively, to geminate recombination and nearly barrierless (based on the time scale) aquation to form ${ }^{1} \mathrm{PtBr}_{5}\left(\mathrm{H}_{2} \mathrm{O}\right)^{-}$, which remains stable at least to $1.2 \mathrm{~ns}$, Fig. 1.
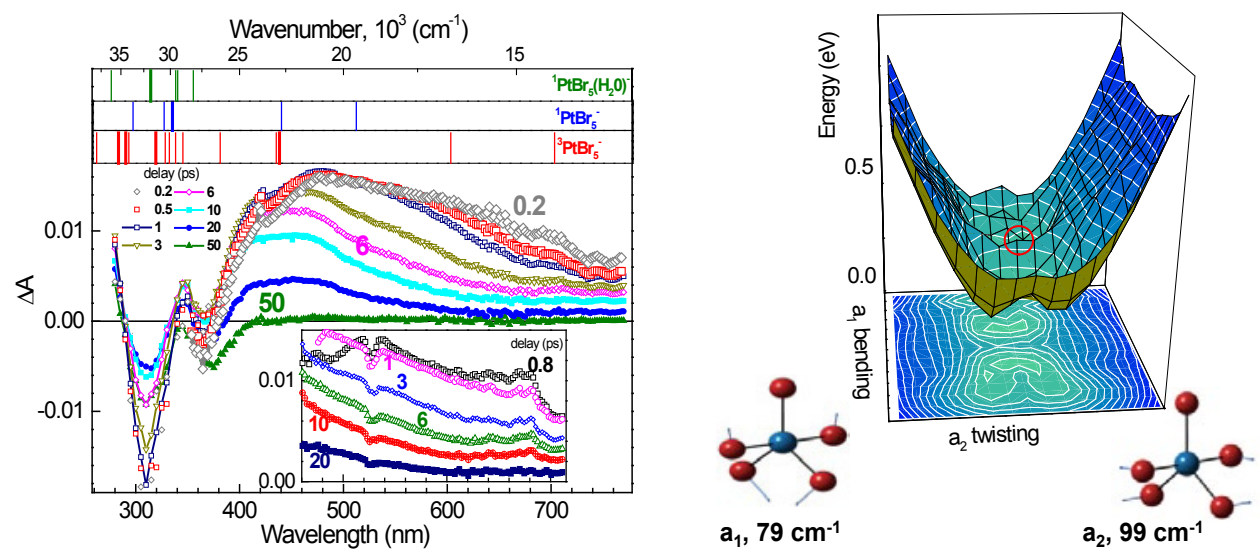

Fig. 1. Left: $420-\mathrm{nm} \Delta \mathrm{A}$ spectra of aqueous $\mathrm{PtBr}_{6}{ }^{2-}$. Bottom inset: $530-\mathrm{nm} \Delta \mathrm{A}$ spectra. Top insets: the TDDFT/M052X/ CPCM VET energies of possible products with oscillator strengths larger (thick lines) and smaller (thin lines) than 0.01. Right: M052X scans of ground-state PESs of ${ }^{3} \mathrm{PtBr}_{5}{ }^{-}$along the "branching space" coordinates. Open circle shows the CI location.

According to our normal mode analysis, the 78 and $114 \mathrm{~cm}^{-1}$ beats are attributed to the bending and umbrella $a_{1}$ modes of ${ }^{3} \mathrm{PtBr}_{5}{ }^{-}$, respectively. The bending $a_{1}$ mode of ${ }^{3} \mathrm{PtBr}_{5}{ }^{-}$corresponds to the reaction coordinate mode parallel to the gradient difference vector of the $\mathrm{JT}_{4 \mathrm{v}} \mathrm{CI}$ present in the photochemical path, Fig. 2. The umbrella $a_{1}$ mode is the "spectator" mode that preserves the electronic degeneracy at the CI. Very rapid (100 fs) loss of coherence in the former case is observed, whereas in the latter case coherence is less damped (time constant, $235 \mathrm{fs}$ ). Following excitation of $\mathrm{PtBr}_{6}{ }^{2-}$ into ${ }^{1} T_{2 \mathrm{~g}}$ (third excited singlet state) ultrafast internal conversion is proposed to lead to the lowest excited ${ }^{\mathrm{P}} T_{1 \mathrm{~g}}$ state, from which the Raman active vibrational modes $\left(\mathrm{t}_{2 \mathrm{~g}}, \mathrm{e}_{\mathrm{g}}\right.$, and $\left.\mathrm{a}_{1 \mathrm{~g}}\right)$ propagate the wavepacket to the ${ }^{3} T_{1 \mathrm{~g}}$ dissociative state. The wavepacket then moves on the ${ }^{3} T_{1 \mathrm{~g}}$ potential energy surface towards the exit, reached without significant spreading very well within the first $150 \mathrm{fs}$ at the nascent $\mathrm{C}_{4 \mathrm{v}}$ geometry of $\mathrm{PtBr}_{5}{ }_{5}^{-}$. In these species, the first excited triplet state of ${ }^{3} E$ character is populated because it correlates to the lowest ${ }^{3} T_{1 \mathrm{~g}}$ state in $\mathrm{O}_{\mathrm{h}}$ point group of $\mathrm{PtBr}_{6}{ }^{2-}$. A degenerate electronic $E$-state at $\mathrm{C}_{4 \mathrm{v}}$ geometry of $\mathrm{PtBr}_{5}{ }^{-}$represents a JT $\mathrm{C}_{4 \mathrm{v}} \mathrm{CI}$ and has its degeneracy split by vibrations of suitable $\left(b_{1}\right.$ and $\left.b_{2}\right)$ symmetry, [4]. When the compact wavepacket passes through the CI, it accelerates on the steep potential slopes of the CI region and the $b_{1}$ and $b_{2}$ splitting modes become excited, where $b_{1}$ pucker in $\mathrm{C}_{4 \mathrm{v}}$ descends to the $a_{1}$ bending mode in $\mathrm{C}_{2 \mathrm{v}}$ observed. The umbrella $a_{1}$ mode can be viewed as the closure of four equatorial ligands on the coordination site vacated by the ligand and is excited due to ligand dissociation.

Fluorescence and transmission x-ray spectra were measured in aqueous solutions of $\mathrm{K}_{2} \mathrm{PtBr}_{6}(5$ $25 \mathrm{mM}$ ) flowed through a $0.5-\mathrm{mm}$ thick jet at (i) $L_{3}\left(2 \mathrm{p}_{3 / 2}\right)$-Pt edge $(11.45-11.75 \mathrm{keV}$ ) and (ii) $K$-Br edge $(13.44-13.55 \mathrm{keV})$ at selected delay times $(0.2,2,20,200$, and $1700 \mathrm{~ns})$ following 100-fs 
400-nm laser pulses $\left(0.15 \mathrm{~mJ}\right.$ pulse $\left.{ }^{-1}\right)$. In addition, differential fluorescence and absorption kinetic traces were measured at 11.562 and $11.567 \mathrm{keV}$ as well as 13.473 and $13.477 \mathrm{keV}$. The bromide ion ligand loss and photoaquation are evidenced through the disappearance and displacement of the $L_{3}\left({ }^{2} \mathrm{p}_{3 / 2}\right)$-Pt and $K$-Br near-edge features observed at $200 \mathrm{ps,} \mathrm{Fig.} 2$. In particular, the XANES signal at the $K$-Br edge mainly consists of a change of the $13.473 \mathrm{keV}$ feature, consistent with a decrease the oxidation state of a bromine atom, [5]. Because of the partially covalent character of $\mathrm{Pt}^{4+}-\mathrm{Br}^{-}$ bond in ground-state $\mathrm{PtBr}_{6}{ }^{2-}$, the decrease of the oxidation state of the $\mathrm{Br}$ atom observed is the evidence for the $\mathrm{Pt}-\mathrm{Br}$ bond rupture in excited $\mathrm{PtBr}_{6}{ }^{2-}$, leading to the formation of a $\mathrm{Br}^{-}$ion. Time scans into a microsecond time domain performed at selected probe energies display an instantaneous rise of the signal at the Pt- and $\mathrm{Br}$-edges followed by decay at the Pt edge. This observation is interpreted as the further decay of the $\mathrm{PtBr}_{5}\left(\mathrm{H}_{2} \mathrm{O}\right)^{-}$complex in which $\mathrm{Pt}$ is reduced to a lower oxidation state.
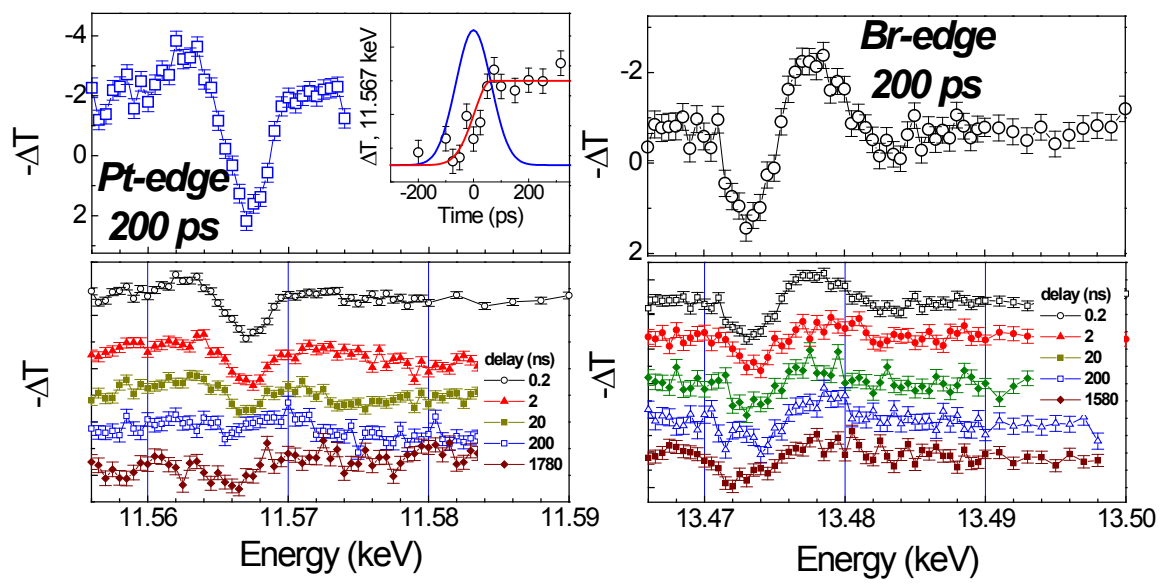

Fig. 2. 400-nm pump/x-ray absorption probe spectra at the Pt- and Br-edges at different time delays shown in legends. The instantaneous signal rise at $11,567 \mathrm{keV}$ is described a step-function convoluted with the Gaussian 150-ps (fwhm) instrument response function (inset).

We acknowledge the NSF support (CAREER CHE-0847707 award: ANT) and an allocation of computer time from the Ohio Supercomputer Center. Time-resolved x-ray absorption experiments were performed on the X05LA beamline at the Swiss Light Source, Paul Scherrer Institut, Villigen, Switzerland.

\section{References}

1. J. Friedrich, S. Glib, O. T. Ehrler, A.Behrendt, and M. M. Kappes, "Electronic Photodissociation spectroscopy of isolated $\operatorname{IrX}_{6}{ }^{2-}(\mathrm{X}=\mathrm{Cl}, \mathrm{Br})$ " J. Chem. Phys. 117, 2635 (2002).

2. I. L. Zheldakov, M. N. Ryazantsev, and A. N. Tarnovsky, "Wavepacket Motion via a Conical Intersection in the Photochemistry of Aqueous Transition-Metal Dianions," J. Phys. Chem. Lett. 2, 1540 (2011).

3. I. P. Pozdnyakov, E. M. Glebov, V. F. Plyusnin, M. V. Tkachenko, and H. Lemmetyinen, "Primary Processes in Photophysics and Photochemistry of $\mathrm{PtBr}_{6}{ }^{2-}$ Complex Studied by Femtosecond Pump-Probe Spectroscopy," Chem. Phys. Lett. 442, 78 (2004).

4. G. A Worth and L. S. Cederbaum "Beyond Born-Oppenheimer: Molecular Dynamics through a Conical Intersection," Annu. Rev. Phys. Chem. 55, 127 (2004).

5. C. G. Elles, I. A. Shkrob, R. A. Crowell, D. A. Arms, and E. C. Landahl, "Transient X- Ray Absorption Spectroscopy of Hydrated Halogen Atom,” J. Chem. Phys. 128, 061102 (2008). 